\title{
¿PUEDE AMORTIGUAR EL ENGAGEMENT LOS EFECTOS NOCIVOS DE LA PROCRASTINACIÓN ACADÉMICA? CAN ENGAGEMENT BUFFER THE HARMFUL EFFECTS OF ACADEMIC PROCRASTINATION?
}

\author{
Marcela Paz González-Brignardello ${ }^{\mathrm{ab}}$ y Ángeles SÁnchez-Elvira-PaniaguA ${ }^{\mathrm{ab}}$ \\ aFacultad de Psicología, Universidad Nacional de Educación a Distancia (UNED).mpgonzalez@psi.uned.es \\ 'Instituto Universitario de Educación a Distancia (IUED), mpgonzalez@psi.uned.es (UNED)
}

Cómo referenciar este artículo/How to reference this article:

González-Brignardello, M. P. y Sánchez-Elvira-Paniagua, A. (2013). ¿Puede amortiguar el engagement los efectos nocivos de la procrastinación académica? [Can Engagement buffer the harmful effects of Academic Procrastination?]. Acción Psicológica, 10(1), 117-134. http://dx.doi.org/10.5944/ap.10.1.7039

\section{Resumen}

El propósito de este estudio es explorar la relación existente entre la Procrastinación Académica y el Engagement, así como el patrón de funcionamiento de ambas variables en entornos académicos, en relación al uso de estrategias de aprendizaje autorregulado, eficientes y deficientes, y el estado de los estudiantes previo a la realización de los exámenes. Todas las variables han sido medidas a través de cuestionarios de autoinforme, en una población de universitarios en modalidad a distancia/en línea. Asimismo, se postula un posible efecto de amortiguación o buffering del Engagement sobre los efectos nocivos de la Procrastinación Académica. Este patrón diferencial se analiza con mayor precisión mediante la consideración de tres perfiles de estudiantes, los caracterizados por Engagement puro, los caracterizados por Procrastinación Académica pura, y un perfil mixto de procrastinadores con niveles de Engagement medio-alto. Los resultados obtenidos corroboran, por un lado, las grandes diferencias existentes en la forma de afrontar los estudios entre los estudiantes engaged y los procrastinadores y, por otro, el claro efecto amortiguador del engagement sobre la procrastinación académica, observándose en el perfil mixto una mejora significativa de carácter positivo, tanto en el tipo de estrategias de aprendizaje autorregulado utilizadas, como en su estado previo a los exámenes.

Palabras clave: Procrastinación Académica; Engagement; estrategias de aprendizaje autorregulado.

\section{Abstract}

The purpose of this study is to explore the relationship between Academic Procrastination and Engagement, as well as the pattern of academic functioning of both variables, regarding the use of self-regulated learning strategies, both efficient and inefficient, as well as students' state previous to the exams. All variables were measured through self-report questionnaires, in a population of distance learning / online university students. Also, a possible buffering effect of Engagement on the harmful effects of academic procrastination is postulat- 
ed. This differential pattern is more accurately analyzed by considering three profiles of students, those characterized by Pure Engagement, those characterized by pure Academic Procrastination and a mixed profile of procrastinators with medium-high Engagement levels. The results corroborate, on the one hand, the large differences that exist in how engaged and procrastinator students cope with their studies and, on the other hand, the clear buffering effect of engagement on academic procrastination that can be observed in the significant and positive improvement showed by the mixed profile both, in the type of self-regulated learning strategies used, and in its prior state to the exams.

Keywords: Academic Procrastination; Engagement; Self-regulated learning strategies.

\section{Introducción}

La realización de una tarea que presenta un límite temporal, la entrega de un trabajo o, incluso, el pago de una obligación tributaria, requieren, no sólo la autorregulación de su proceso de realización, sino también el cumplimiento de unos plazos temporales que delimitan la acción. Las personas se diferencian en el comportamiento que presentan ante dichos deadlines y esas diferencias, en caso de ser comportamientos habituales, tienen consecuencias que determinan aspectos, no solo de rendimiento, sino también de percepción de autoeficacia, estrés y bienestar, entre otros.

La tendencia a retrasar intencionalmente el inicio o la terminación de actividades, más allá de los plazos temporales fijados o deadlines, se denomina Procrastinación (Ariely y Wertenbroch, 2002; Howell, Watson, Powell y Buro, 2006; Lay y Silverman, 1996). Este retraso intencional se realiza a pesar de que las personas sean conscientes de que, dicha demora, les pueda generar una disminución de su bienestar y, más aún, un incremento del malestar psicológico (Steel, 2007) "hasta el punto de la incomodidad subjetiva» (Ferrari, Johnson y McCown, 1995, citado en Ferrari, 2010, p. 15). Para Solomon y Rothblum (1984), se trata de «el acto de retrasar innecesariamente la realización de tareas hasta el punto de experimentar incomodidad subjetiva» (p. 503).

La Procrastinación es un comportamiento muy prevalente en la población general. Entre el $15 \%$ y el $20 \%$ de la población adulta presenta procrastinación crónica, igualmente repartida entre ambos sexos (e.g., Harriott y Ferrari, 1996). No obstante, en un estudio de metaanálisis, Van Eerde (2003) encontró mayor Procrastinación en los hombres que en las mujeres. Por otro lado, estudios realizados en la población general muestran que entre el 20\% y el 30\% de las personas consideran que la procrastinación es un problema importante en sus vidas y que les genera problemas personales, laborales y económicos (e.g., Ferrari, Johnson y McCown, 1995, citado en Ferrari, 2010).

Desde el ámbito de la investigación psicológica, la procrastinación se ha relacionado con ansiedad (e.g., Rosário et al., 2008; Rothblum, Solomon y Murakami, 1986), depresión (e.g., Beswick, Rothblum y Mann, 1988), tensión o estrés (e.g., Beswick et al., 1988; Macan, 1994), baja autoeficacia (e.g., Ferrari, Parker y Ware, 1992) y baja autoestima (e.g., Beswick et al., 1988). En cuanto a las áreas de rendimiento, se han encontrado relaciones significativas con disminución del rendimiento académico (Rothblum et al., 1986) y laboral (Macan, 1994).

Desde una perspectiva motivacional, la procrastinación ha sido entendida como un fallo en el proceso de autorregulación (e.g., Steel, 2007; Wolters, 2003). Otros autores la han considerado como un mecanismo protector de la autoestima, debido al miedo al fracaso y, por lo tanto, como una estrategia de self-handicapping (e.g., Ferrari y Tice, 2000). Estos resultados son debidos a que la procrastinación no se explica solamente como consecuencia de una mala planificación del tiempo, sino que consiste en una compleja interacción entre componentes afectivos, cognitivos y conductuales, lo que da paso a una gran diversidad de manifestaciones.

Un tipo específico de procrastinación, de especial relevancia, es el que se produce en población estudiantil, denominado Procrastina- 
ción Académica. Su prevalencia es aún mayor que la de la procrastinación general, encontrándose entre el 30\% y el $60 \%$ en estudiantes (Özer, Demir y Ferrari, 2009; Pychyl, Lee, Thibodeau, y Blunt, 2000; Solomon y Rothblum, 1984; Steel, 2007). En cuanto a la evolución de la procrastinación académica con la edad, los datos de la investigación se muestran inconsistentes, ya que se han referido estudios donde se observa un aumento de ella con la edad (e.g., Semb, Glick y Spencer, 1979) y también otros en los cuales disminuye (e.g., Beswick et al., 1988).

A su vez, Solomon y Rothblum (1984) encontraron dos tipos de procrastinadores en el ámbito académico: un grupo cuya principal característica es el miedo al fracaso (ansiedad ante la evaluación, baja autoconfianza y alto perfeccionismo), y otro grupo heterogéneo formado por lo que los autores llamaron aversión a la tarea (mal manejo del tiempo y dificultad para la toma de decisiones). Del mismo modo, también se ha observado que no se pospone o retrasa el cumplimiento de todas las tareas académicas por igual (e.g., Blunt y Pychyl, 2000).

A la luz de los resultados obtenidos hasta el momento, parece necesario encontrar fórmulas que ayuden a paliar los efectos negativos de la procrastinación. De especial interés es entender y ayudar a prevenir la Procrastinación Académica, debido al riesgo de abandono y fracaso que comporta para los estudiantes. En este sentido, una característica personal cuya investigación está experimentando un notable auge en los últimos años, y que podría pensarse que tuviese efectos contrarios a la procrastinación, es el Engagement. El Engagement se define como «un estado mental positivo con el trabajo y caracterizado por vigor, dedicación y absorción» (Schaufeli, Salanova, González-Romá y Bakker, 2002, p. 79). El Engagement comporta tres dimensiones distintas: el Vigor, definido por altos niveles de energía, esfuerzo y persistencia en el trabajo; la Dedicación, que consiste en proporcionar un gran significado, entusiasmo y valoración al trabajo; y, finalmente, la Absorción, caracterizada por un estado de concentración plena y felicidad al trabajar, y de tener la sensación de que el tiempo «pasa volando» (Salanova, Schaufeli, Llorens, Peiró y Grau, 2000). Esta última dimensión sería similar al concepto de Flow propuesto por Csikszentmihalyi (1990), aunque el flow se refiera más a la experiencia a corto plazo que a un estado persistente de la mente, como ocurre en el caso del Engagement (Schaufelli, Salanova et al., 2002).

La definición anteriormente expuesta proviene del ámbito de la Psicología Positiva, cuyo objetivo es estudiar las fortalezas y el funcionamiento óptimo del ser humano, más que sus debilidades y disfunciones (Peterson y Seligman, 2004; Seligman y Csikszentmihalyi, 2000). El Engagement responde, por tanto, a un concepto motivacional positivo relacionado con el trabajo y la vida, y es concebido como un estado psicológico duradero en el tiempo (Salanova et al., 2000). Este constructo ha sido ampliamente investigado en ámbitos laborales (Salanova et al., 2000; Schaufeli, Salanova et al., 2002) como variable opuesta al Burnout (Freuderberger, 1974). Actualmente, su estudio se ha extendido al entorno académico (Martínez y Salanova, 2003; Schaufeli, Martínez, Marques, Salanova y Bakker, 2002), y podría representar una de las fórmulas de prevención o amortiguación de la procrastinación aún no explorada.

En el ámbito educativo, el Engagement no es un constructo nuevo; tiene una larga tradición en la investigación psicológica y educativa cuya finalidad es la mejora del rendimiento académico de los estudiantes (Reschly y Christerson, 2012). Ha sido definido de distintas formas y eso se traduce, en ocasiones, en una ausencia de claridad conceptual. No obstante, en lo que la mayoría de los investigadores están de acuerdo es en su carácter complejo y multidimensional (Christenson, Reschly y Wylie, 2012). En todo caso, está ampliamente demostrado el efecto beneficioso del Engagement para los estudiantes, tanto desde el punto de vista del rendimiento académico como de su bienestar. Así, los estudiantes engaged utilizan estrategias más efectivas en su proceso de aprendizaje relacionadas con estrategias de autorregulación bien conocidas como son el establecimiento de objetivos y metas, la planificación, la organización y la monitorización de 
sus estudios. Al tiempo, estos estudiantes también se esfuerzan, persisten, se retan a si mismos y disfrutan de estos retos y del propio proceso de aprendizaje (Brooks, Brooks y Godstein, 2012).

Llegados a este punto, cabe preguntarse, en primer lugar, si la Procrastinación Académica y el Engagement pueden estar inversamente relacionados y, en segundo lugar si, de ser así, el Engagement podría producir un efecto de amortiguación o buffering sobre los efectos negativos de la procrastinación en entornos académicos. Este efecto podría estar vinculado, desde una óptica procesual, al tipo de estrategias específicas de autorregulación del aprendizaje puestas en marcha por los estudiantes, en función de estas dos importantes características personales (Park y Sperling, 2012).

Para llevar a cabo una exploración inicial de estos supuestos se realizó este estudio, en el marco de la V convocatoria de Redes de Investigación en Innovación Docente, convocada por el vicerrectorado de Coordinación, Calidad e Innovación de la UNED. El presente estudio se integra en las investigaciones realizadas en la Red de Aprendizaje Óptimo, Engáncha-TE, de carácter institucional, que se enmarca en el Plan de Acogida de la UNED; en concreto, en el Plan de Acogida Virtual (PAV). Desde 2006, todos los estudiantes de nueva matrícula en la UNED son dados de alta en la Comunidad de Acogida Virtual de su Facultad o Escuela, cuyo propósito es el apoyo y orientación de los estudiantes nuevos durante su primer año en la universidad y, en última instancia, la prevención del abandono (Sánchez-Elvira-Paniagua, González-Brignardello y Santamaría-Lancho, 2009). La finalidad de la RED de innovación consistió en estudiar aquellas características personales que dificultan o promueven el bienestar y rendimiento estudiantil. Dentro de este marco general se inserta el presente estudio, cuyos objetivos específicos son dar respuesta a las siguientes cuestiones:

1. ¿Están la Procrastinación y el Engagement académicos inversamente relacionados?

2. ¿De qué modo se relacionan ambas características personales con estrategias de aprendizaje y estudio autorregulados, tanto positivas como negativas; $y$, finalmente.

3. ¿Ejerce el Engagement académico un efecto buffering, o de amortiguación, sobre las consecuencias negativas de la Procrastinación académica?

De ser así, los resultados obtenidos podrán ser interesantes para el desarrollo de programas de intervención y prevención del abandono y el fracaso estudiantil.

\section{Método}

\section{Participantes}

Un total de 9290 estudiantes nuevos realizó voluntariamente alguna/s de la/s fases de recogida de información. De este total, 415 firmaron un contrato inicial y cumplimentaron el total de cuestionarios de la RED Engáncha-TE. La depuración de los valores perdidos de la muestra inicial concluyó en una muestra final de 377 estudiantes pertenecientes a las nueve Facultades y dos Escuelas Superiores de Ingenieros de la UNED; 173 hombres (45.9\%) y 204 mujeres $(54.1 \%)$ con una media de edad de 34.25 años (DT $=8.62)$ en un rango de edad comprendido entre los 18 y 58 años. La muestra es representativa del alumnado habitual de la UNED.

\section{Instrumentos de evaluación}

Los estudiantes respondieron, durante diferentes fases, a una amplia batería de cuestionarios en línea. A efectos del presente estudio, se tomaron en consideración los siguientes instrumentos:

\section{- Procrastinación y Engagement:}

Procrastinación Académica (González-Brignardello y Sánchez-Elvira-Paniagua, Cuestionario Experimental). Cuestionario diseñado para evaluar conducta de Procrastinación en ámbito académico. Consta de 18 ítems y escala Likert de 5 puntos sobre grado de acuerdo 
de 1 = Nada, en absoluto a 5 = Totalmente. Un ejemplo de ítem es: "Habitualmente pospongo estudiar porque pienso que aún me queda mucho tiempo para hacerlo». Este cuestionario se basó en una traducción de la Escala de Procrastinación (Bruinsma y Jansen, 2009) compuesta por 8 ítems, y en ítems creados a partir de información recogida en las Comunidades Virtuales, en relación al modo de afrontar el proceso de estudio autónomo y sus problemas.

Engagement (Salanova et al., 2000, adaptación para estudiantes). Mide tres dimensiones: Vigor, Dedicación y Absorción, cada una formada por 5 ítems en escala Likert de 7 puntos, $(0=$ Nunca a $6=$ Siempre $)$. Un ejemplo de ítem es: "Olvido todo lo que pasa alrededor de mí cuando estoy abstraído con mis estudios». Se obtuvo el valor de Engagement total: suma de los valores de las 3 dimensiones, dividido por el número de dimensiones.

- Estrategias de aprendizaje autorregulado y hábitos de estudio:

Batería de estrategias y hábitos de estudio (Sánchez-Elvira-Paniagua, Fernández y Amor, 2006a) compuesta por un total de 8 subescalas derivadas de un análisis factorial realizado en una muestra de 49337 estudiantes pertenecientes a todas las Facultades y Escuelas de la UNED: (a) Manejo ineficaz del tiempo (13 ítems); (b) Ansiedad (8 ítems); (c) Persistencia (7 ítems); (d) Procesamiento profundo (6 ítems); (e) Procesamiento superficial (6 ítems); (f) Organización de la información (5 ítems); (g) Evitación de dificultades (4 ítems); y (h) Pautas fijas de estudio (3 ítems). La escala de respuesta es tipo Likert de 5 puntos, ( $1=$ Nada, en absoluto y 5 = Totalmente).

Asimismo, esta batería permite obtener dos factores globales de segundo orden: (a) Autorregulación eficiente del aprendizaje constituido por Persistencia, Procesamiento profundo y Procesamiento superficial, Organización de la información, Estudio compartido y Pautas fijas de estudio; y (b) Autorregulación deficiente del aprendizaje constituido por Manejo ineficaz del tiempo, Ansiedad, Evitación de las dificultades y Procesamiento superficial (Sánchez-Elvira-Paniagua, Fernández y Amor, 2006b).
- Percepción de preparación, atribuciones y estados durante la fase previa a los exámenes del primer semestre:

Batería experimental elaborada ad hoc para el Plan de Acogida Virtual UNED - Fase de preparación de exámenes (Sánchez-Elvira-Paniagua y González-Brignardello, Cuestionario Experimental). Se utilizaron únicamente los factores obtenidos con los ítems relativos a la fase previa a los exámenes: estados de ánimo más frecuentes, tipo de interferencias sufridas y dificultades encontradas durante el proceso de estudio, y grado percibido de preparación para los exámenes.

- Dificultades atribuidas a falta de tiempo (3 ítems), e.g., "responsabilidades familiares».

- Interferencias por falta de hábitos de estudio (5 ítems), e.g., «me he distraído fácilmente mientras estudio».

- Percepción de buena preparación para los exámenes (4 ítems), e.g., "creo que, por lo general, llevo bien preparadas las asignaturas».

- Estado previo a exámenes: estado de ánimo Ansioso (6 ítems), e.g., "preocupación»; estado de ánimo Positivo (5 ítems), e.g., "entusiasmo»; Estado somático Corporal (2 ítems), e.g., "cansancio»; y Desinterés (3 ítems), e.g., «aburrimiento».

Todas las preguntas se presentaron en formato de respuesta tipo Likert de 5 puntos, donde el grado de acuerdo oscilaba de 1 = Nada, en absoluto a $5=$ Totalmente.

\section{Procedimiento}

Al comenzar el curso académico, todos los estudiantes nuevos fueron dados de alta en la Comunidad de Acogida Virtual correspondiente; en la plataforma virtual educativa de la UNED (aLF). En estas Comunidades, los estudiantes disponen de contenidos y actividades secuenciadas, que responden a las etapas de integración propias del primer año de matriculación. Dichas comunidades son coordinadas 
por un/a profesor/a de cada Facultad/Escuela y se favorece el apoyo entre pares.

Al principio del curso académico, se realizó una invitación en línea a participar en la RED de innovación y se ofreció 1 ECTS de libre configuración a quienes realizasen todas las actividades de la Red. A lo largo del primer semestre se fueron habilitando los cuestionarios, todos para su cumplimentación en línea de acuerdo a las siguientes fases:

- Fase de inicio/integración: contrato de participación y cuestionario de identificación/variables socioculturales. Se mantuvo activo durante el mes de noviembre.

- Fase de estudio: cuestionarios de Procrastinación Académica, Engagement y Estrategias y Hábitos de Estudio. Estuvieron activos durante el mes de diciembre.

- Fase previa a los exámenes: batería experimental del PAV, disponible a partir de la semana previa a los exámenes.

Para realizar el estudio comparativo y analizar el posible efecto de buffering del Engagement sobre la Procrastinación Académica, se formaron grupos extremos a partir de las pun- tuaciones de los participantes en estas dos escalas, con el objetivo de combinar los niveles de procrastinación y engagement de los estudiantes, obteniendo tres perfiles:

- Perfil 1 o Engagement puro (P1): alto en Engagement $(\mathrm{Pc}>$.70) y medio-bajo en Procrastinación (menor o igual a la media en Procrastinación).

- Perfil 2 o Procrastinadores con Engagement (P2): alto en Procrastinación (Pc > .70) y medio-alto engagement (mayor o igual a la media en Engagement).

- Perfil 3 o Procrastinadores puros (P3): alto en Procrastinación ( $\mathrm{Pc}>.70)$ y medio-bajo Engagement (menor o igual a la media en Engagement).

Se comprobó la correcta configuración de los tres perfiles, es decir, que diferenciasen claramente los tipos de estudiantes en función de los propósitos de la investigación, a través de análisis de varianza de un factor de medidas independientes, Perfil, sobre las variables dependientes Procrastinación y Engagement (las variables de clasificación), con tres niveles (P1, P2 y P3). En la Tabla 1 puede verse el resultado de los ANOVAs, los contrastes post-hoc y el tamaño del efecto (ver tabla 1).

\section{Tabla 1}

Medias, desviaciones típicas, resultados del análisis de varianza y tamaño del efecto para los tres perfiles en las variables de clasificación

\begin{tabular}{lcccccccc}
\hline & $\begin{array}{c}\text { Perfil 1 } \\
\mathbf{n = 8 9}\end{array}$ & $\begin{array}{c}\text { Perfil 2 } \\
\mathbf{n}=\mathbf{3 7}\end{array}$ & $\begin{array}{c}\text { Perfil 3 } \\
\mathbf{n}=\mathbf{7 2}\end{array}$ & & $\mathbf{n}_{\mathrm{p}}^{2}$ & \multicolumn{3}{c}{$\begin{array}{c}\text { Tamaño del efecto } \\
\text { g de Hedges }\end{array}$} \\
\hline $\mathrm{M}(\mathrm{DT})$ & $\mathrm{M}(\mathrm{DT})$ & $\mathrm{M}(\mathrm{DT})$ & $\mathrm{F}(2,195)$ & & $\mathrm{P} 1 / \mathrm{P} 2$ & $\mathrm{P} 1 / \mathrm{P} 3$ & $\mathrm{P} 2 / \mathrm{P} 3$ \\
\hline Procrast. & $1.70_{\mathrm{a}}(.35)$ & $3.04_{\mathrm{b}}(.37)$ & $3.12_{\mathrm{b}}(.44)$ & $313.14^{* *}$ & .76 & -3.74 & -3.60 & -.19 \\
Académica & & & & & & & & \\
Engagement & $5.27_{\mathrm{a}}(.29)$ & $4.98_{\mathrm{b}}(.42)$ & $3.67_{\mathrm{c}}(.57)$ & $286.87^{* *}$ & .75 & .87 & 3.64 & 2.48 \\
\hline
\end{tabular}

Nota: Procrast. (Procrastinación). P1/P2, P2/P3 y P1/P3 (comparaciones entre perfiles 1, 2 y 3).

Dentro de cada fila las medias con diferentes subíndices difieren al nivel de $p<.05$. ${ }^{* *} p=.000$ 
- Los dos grupos de procrastinadores mostraron niveles similares en la variable Procrastinación. El tamaño del efecto de las diferencias de medias entre el perfil de Engagement puro con los dos grupos de procrastinadores es muy grande (Rosenthal, 1996).

- Los niveles de Engagement difirieron significativamente entre los tres perfiles, observándose un incremento progresivo desde el P3 al P1. El tamaño del efecto de las diferencias post-hoc entre los grupos de Engagement puro y Procrastinación pura es muy grande, mientras que el tamaño del efecto entre los procrastinadores con Engagement medio alto y el grupo de Engagement Puro, es grande (Cohen, 1988; Rosenthal, 1996).

\section{Diseño y análisis de datos}

Se utilizó un diseño ex post-facto prospectivo factorial con muestreo no aleatorizado, de carácter incidental (Montero y León, 2007).

Para la contrastación de las hipótesis se llevaron a cabo, en primer lugar, análisis descriptivos, en segundo lugar análisis de correlación mediante el estadístico de Pearson y, finalmente, análisis de varianza de un factor de medidas independientes con tres niveles. Los ANOVAs se realizaron para el estudio del efecto de la variable independiente de clasificación generada, Perfil, con tres niveles, sobre las variables dependientes relativas a las estrategias de aprendizaje autorregulado utilizado durante los meses de estudio, y las variables de estado previo a la realización de los exámenes, tal y como se detalla en el apartado de resultados. Previamente, se comprobaron los supuestos de normalidad y homocedasticidad de las variables consideradas. Dependiendo del resultado de la prueba de homogeneidad de varianzas, para realizar las comparaciones múltiples post hoc, se aplicó la prueba de Scheffé (asumiendo igualdad de varianzas) o Games-Howell (no asumiendo igualdad de varianzas).

Se calculó, asimismo, el tamaño del efecto en todos los análisis realizados. En las correlaciones se empleó la $d$ de Cohen, en los ANOVAs la eta cuadrado parcial y, por su interés para un análisis pormenorizado, se incluyó asimismo el tamaño del efecto de los post-hoc, mediante la $g$ de Hedges (Ellis, 2009).

\section{Resultados}

En la tabla 1 del Apéndice se presentan los descriptivos de las diferentes variables estudiadas, agrupadas según el instrumento que las recoge y la fase en la cual se han presentado. Las medias de cada variable se corresponden con la transformación de la puntuación directa según la escala Likert utilizada (ver apartado instrumentos).

Respondiendo a la primera pregunta planteada, ambas variables, Procrastinación Académica y Engagement, se relacionaron inversamente y de manera significativa $(r=-.34, p=$ $0.01, d=-.72)$. A su vez, las dimensiones del Engagement, Vigor y Absorción, correlacionaron también significativa y negativamente con la Procrastinación Académica ( $r=-.359$ y $r=$ -.340, $p=0.01, d=-.77)$. La dimensión de Dedicación no se relacionó con la Procrastinación Académica de forma significativa.

Para responder a la segunda pregunta, se analizaron las relaciones entre ambas variables y el resto de las variables del estudio obteniéndose los resultados que se muestran en la tabla 2 (únicamente se muestran las $r>.30$ ). Como puede apreciarse, el patrón de relaciones de ambos constructos es inverso. 
Tabla 2

Correlaciones y tamaño del efecto del Engagement y la Procrastinación Académica con las variables objeto de estudio

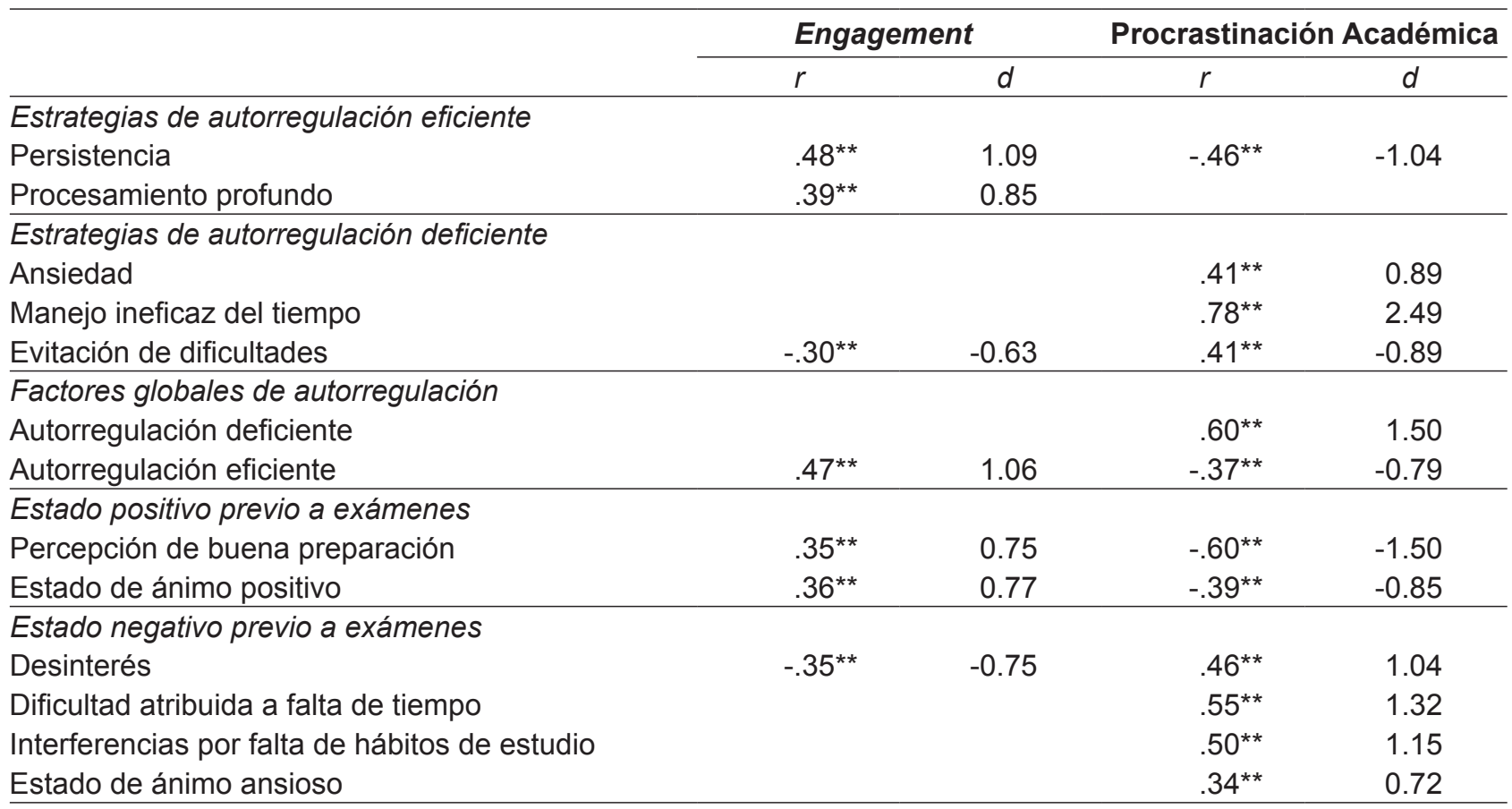

Nota. $* * p<.01$

Para analizar el posible efecto de amortiguación, o buffering, se procedió a analizar, en primer lugar, el uso diferencial de estrategias y hábitos de aprendizaje autorregulado globales (eficientes y deficientes) entre los tres perfiles (P1, P2 y P3), durante la fase de estudio. El resultado de dicho análisis puede verse en la tabla 3 .

Tabla 3

Medias, desviaciones típicas, resultados de los ANOVAs y tamaño del efecto para los tres perfiles, en el uso estrategias globales de autorregulación del aprendizaje

\begin{tabular}{|c|c|c|c|c|c|c|c|c|}
\hline & $\begin{array}{c}\text { Perfil } 1 \\
n=89\end{array}$ & $\begin{array}{c}\text { Perfil } 2 \\
n=37\end{array}$ & $\begin{array}{c}\text { Perfil } 3 \\
n=72\end{array}$ & & $\eta_{\mathrm{p}}^{2}$ & $\begin{array}{r}\text { Tam } \\
\mathrm{g}\end{array}$ & $\begin{array}{l}\text { ño del } \\
\text { le Heds }\end{array}$ & $\begin{array}{l}\text { fecto } \\
\text { es }\end{array}$ \\
\hline & $\mathrm{M}(\mathrm{DT})$ & M (DT) & $\mathrm{M}(\mathrm{DT})$ & $F(2,195)$ & & $\mathrm{P} 1 / \mathrm{P} 2$ & P1/P3 & P2/P3 \\
\hline $\begin{array}{l}\text { Autorreg. } \\
\text { Eficiente }\end{array}$ & $17.14_{a}(1.56)$ & $16.26_{b}(1.69)$ & $14.73_{c}(1.77)$ & $41.83^{* *}$ & .30 & 0.55 & 1.45 & 0.87 \\
\hline $\begin{array}{l}\text { Autorreg. } \\
\text { Deficiente }\end{array}$ & $7.74_{a}(1.09)$ & $9.30_{b}(1.33)$ & $9.60_{b}(1.58)$ & $88.17^{* *}$ & .31 & -1.33 & -1.39 & -0.20 \\
\hline
\end{tabular}

Nota. Autorreg. (Autorregulación).

** Significación $=.00$. Post hoc: dentro de cada fila las medias con diferentes subíndices difieren al nivel de $p<.05$.

La tabla 3 muestra la dirección significativamente contraria que siguen los perfiles en cuanto a su puntuación en las estrategias de autorregulación del aprendizaje globalmente consideradas, eficientes y deficientes. El análisis del tamaño del efecto aporta información 
muy valiosa acerca de las diferencias entre los distintos perfiles. Siguiendo la interpretación de estos índices propuesta por Cohen (1988), las $d$ entre 0.50 y 0.79 estarían indicando un efecto moderado, y grande por encima de 0.80 . Apoyándonos en Rosenthal (1996), a partir de 1.30 podría considerarse un efecto muy grande.

Posteriormente, y a la vista de los resultados, se procedió a analizar el uso de las distin- tas estrategias de autorregulación incluidas en los dos factores globales (eficiente y deficiente), consideradas, esta vez, de forma independiente.

En la tabla 4 se muestran las diferencias en el uso de estrategias y hábitos de autorregulación, tanto eficientes como ineficientes, por perfiles.

Tabla 4

Medias, desviaciones típicas, resultados de los ANOVAs y tamaño del efecto para los tres perfiles en el uso de estrategias y hábitos eficientes e ineficientes de aprendizaje

\begin{tabular}{|c|c|c|c|c|c|c|c|c|}
\hline & \multirow{2}{*}{$\begin{array}{c}\text { Perfil } 1 \\
\mathbf{n}=\mathbf{8 9} \\
M(D T)\end{array}$} & \multirow{2}{*}{$\begin{array}{c}\text { Perfil } 2 \\
\mathbf{n}=37 \\
M(D T)\end{array}$} & \multicolumn{2}{|l|}{$\begin{array}{c}\text { Perfil } 3 \\
n=72\end{array}$} & \multirow[t]{2}{*}{$\mathbf{\eta}_{\mathrm{p}}^{2}$} & \multicolumn{3}{|c|}{$\begin{array}{l}\text { Tamaño del efecto } \\
\text { g de Hedges }\end{array}$} \\
\hline & & & $\mathrm{M}(\mathrm{DT})$ & $\mathrm{F}(2,195)$ & & $\mathrm{P} 1 / \mathrm{P} 2$ & $\mathrm{P} 1 / \mathrm{P} 3$ & P2/P3 \\
\hline \multicolumn{9}{|c|}{ Estrategias eficientes } \\
\hline Persistencia & $3.74_{a}(.52)$ & $3.49_{b}(.38)$ & $3.07_{c}(.29)$ & $62.10^{* *}$ & .39 & 0.51 & 1.54 & 1.24 \\
\hline $\begin{array}{l}\text { Procesam. } \\
\text { Profundo }\end{array}$ & $3.19_{a}(.46)$ & $3.10_{a}(.56)$ & $2.68_{b}(.52)$ & $21.71^{* *}$ & .18 & 0.18 & 1.04 & 0.78 \\
\hline $\begin{array}{l}\text { Pauta fijas } \\
\text { de estudio }\end{array}$ & $3.77_{a}(.42)$ & $3.50_{b}(.64)$ & $3.26_{b}(.61)$ & $17.75^{* *}$ & .15 & 0.54 & 0.99 & 0.38 \\
\hline $\begin{array}{l}\text { Organiz. de la } \\
\text { Información }\end{array}$ & $3.46_{a}(.56)$ & $3.12_{b}(.78)$ & $2.94_{b}(.66)$ & $13.42^{* *}$ & .12 & 0.53 & 0.85 & 0.25 \\
\hline $\begin{array}{l}\text { Procesam. } \\
\text { Superficial }\end{array}$ & $2.98_{\mathrm{ab}}(.65)$ & $3.06_{a}(.59)$ & $2.78_{b}(.52)$ & $3.50^{*}$ & .04 & 0.33 & -0.12 & 0.51 \\
\hline \multicolumn{9}{|c|}{ Estrategias ineficientes } \\
\hline $\begin{array}{l}\text { Manejo } \\
\text { Ineficaz del } \\
\text { Tiempo }\end{array}$ & $1.77_{a}(.42)$ & $2.59_{b}(.52)$ & $2.81_{c}(.45)$ & $115.11^{* *}$ & .54 & -1.80 & -2.39 & -0.46 \\
\hline $\begin{array}{l}\text { Evitación de } \\
\text { Dificultades }\end{array}$ & $1.30_{a}(.31)$ & $1.49_{b}(.50)$ & $1.80_{b}(.52)$ & $26.82^{* *}$ & .21 & -0.50 & -1.19 & -0.60 \\
\hline $\begin{array}{l}\text { Ansiedad } \\
\text { ante estudios }\end{array}$ & $1.70_{a}(.52)$ & $2.17_{b}(.75)$ & $2.22_{b}(.76)$ & $14.23^{* *}$ & .13 & -0.78 & -0.81 & -0.06 \\
\hline
\end{tabular}

Nota. Procesam. (Procesamiento); Organiz. (Organización).

Dentro de cada fila las medias con diferentes subíndices difieren al nivel de $p<.05$.

$* p<.05, * * p=.000$

Hemos clasificado la variable procesamiento superficial dentro de las estrategias eficientes; si bien los datos de estudios previos muestran que es una estrategia utilizada por ambos perfiles de autorregulación, eficientes y deficientes, con la diferencia de que en el caso de los primeros, los es- tudiantes hacen uso de ambas, profundas y superficiales (Sánchez-Elvira-Paniagua et al., 2006b).

Los análisis realizados con las estrategias y hábitos eficientes mostraron que el Perfil de Engagement puro presentó los valores más altos en la utilización de este tipo de estrategias, así 
como de los hábitos de estudio favorecedores del aprendizaje, siendo la Persistencia la estrategia más diferenciadora entre los tres perfiles.

Por otra parte, el análisis del uso de estrategias de autorregulación del aprendizaje deficientes o entorpecedoras del aprendizaje reveló, asimismo, diferencias claras entre los perfiles, observándose en el Perfil de Procrastinadores Puros, o Perfil 3, los valores más altos en el Manejo ineficaz del tiempo y la Evitación de las dificultades, siendo la primera la variable más diferenciadora entre los tres perfiles.

En estos análisis, el estudio detallado de los efectos post-hoc, y particularmente del tamaño del efecto, permitió contrastar la hipótesis relativa al efecto del Engagement sobre la Procrasti- nación mediante las comparaciones entre los dos perfiles de procrastinadores, -P2 y P3-, pudiéndose observar claros efectos de amortiguación en prácticamente todas las variables consideradas.

Una vez analizadas las diferencias presentadas en la fase de estudio, se procedió a contrastar aquellas variables recogidas durante la fase previa a los exámenes, es decir, la Percepción de estar bien preparados para presentarse a exámenes, y el estado en el cual se encontraban de manera más habitual durante ese período.

La tabla 5 presenta los resultados de esta comparación, una vez organizadas las variables dependientes en función de la cualidad del estado previo: positivo o negativo.

Tabla 5

Medias, desviaciones típicas, resultados de los ANOVAs y tamaño del efecto para los tres perfiles, en las variables relacionadas con estado positivo y estado negativo previo a los exámenes

\begin{tabular}{|c|c|c|c|c|c|c|c|c|}
\hline & \multirow{2}{*}{$\begin{array}{c}\begin{array}{c}\text { Perfil } 1 \\
\mathbf{n}=89\end{array} \\
M(D T)\end{array}$} & \multirow{2}{*}{ 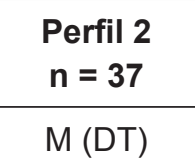 } & \multicolumn{2}{|l|}{$\begin{array}{c}\text { Perfil } 3 \\
n=72\end{array}$} & \multirow[t]{2}{*}{$\eta_{\mathrm{p}}^{2}$} & \multicolumn{3}{|c|}{$\begin{array}{l}\text { Tamaño del efecto } \\
\text { g de Hedges }\end{array}$} \\
\hline & & & $\mathrm{M}(\mathrm{DT})$ & $F(2,195)$ & & $\mathrm{P} 1 / \mathrm{P} 2$ & $\mathrm{P} 1 / \mathrm{P} 3$ & $\mathrm{P} 2 / \mathrm{P} 3$ \\
\hline \multicolumn{9}{|l|}{ Estado positivo } \\
\hline $\begin{array}{l}\text { Percep. Buena } \\
\text { Preparación }\end{array}$ & $3.41_{a}(.56)$ & $2.72_{b}(.55)$ & $2.27_{c}(.57)$ & $82.79^{* *}$ & .46 & 1.23 & 2.01 & 0.79 \\
\hline $\begin{array}{l}\text { Estado de } \\
\text { ánimo Positivo }\end{array}$ & $3.25_{a}(.85)$ & $2.83_{b}(.76)$ & $2.21_{c}(.52)$ & $40.67^{* *}$ & .03 & 0.51 & 1.43 & 1.01 \\
\hline \multicolumn{9}{|l|}{ Estado negativo } \\
\hline $\begin{array}{l}\text { Dificultad atrib. } \\
\text { falta de tiempo }\end{array}$ & $2.82_{a}(.97)$ & $3.35_{b}(.63)$ & $2.84_{a b}(.69)$ & $38.06^{* *}$ & .28 & -1.20 & -0.72 & -0.13 \\
\hline $\begin{array}{l}\text { Interf por falta } \\
\text { de hábitos de } \\
\text { estudio }\end{array}$ & $1.80_{a}(.76)$ & $2.57_{b}(1.08)$ & $2.88_{b}(1.15)$ & $25.28^{* *}$ & .21 & -0.88 & -1.13 & -0.27 \\
\hline Desinterés & $1.12_{\mathrm{a}}(.34)$ & $1.40_{b}(.48)$ & $1.76_{c}(.61)$ & $117.75^{\star *}$ & .26 & -0.72 & -1.33 & -0.63 \\
\hline $\begin{array}{l}\text { Estado de } \\
\text { ánimo ansioso }\end{array}$ & $2.05_{a}(.80)$ & $2.48_{b}(.81)$ & $2.57_{b}(.85)$ & $8.91^{* *}$ & .13 & -0.53 & -0.63 & -0.11 \\
\hline
\end{tabular}

Nota. Percep. (Percepción) Atrib. (Atribución); Interf. (Interferencias)

$* * p=.00$ Post hoc: dentro de cada fila, las medias con diferentes subíndices difieren al nivel de $p<0.05$ 
El análisis del estado de ánimo referido y de la percepción que los estudiantes tienen de su preparación en el período previo a los exámenes mostró, igualmente, diferencias entre los tres perfiles en relación a las variables relativas a un estado positivo, como a las relacionadas con un estado negativo previo a los exámenes.

De forma resumida, el análisis de los efectos post-hoc indica lo siguiente:

- Estado positivo: el Perfil 1, Engagement puro, presentó los niveles más altos de Percepción de buena preparación para los exámenes, así como de Estado de ánimo positivo de los estudiantes, siendo éstas las dos variables más diferenciadoras entre los tres perfiles. Por otro lado, los procrastinadores con Engagement medio-elevado, Perfil 2, mostraron estar en un mejor estado que los procrastinadores puros, antes de los exámenes.

- Estado negativo: el Perfil 3, procrastinadores puros, mostró los mayores niveles de Desinterés, diferenciándose los tres perfiles entre sí. En el resto de variables de este bloque, el patrón de resultados fue idéntico, los perfiles dos y tres, o perfiles de procrastinadores, no difirieron entre sí, presentando niveles más elevados de Atribuciones a la falta de tiempo, Interferencias por falta de hábitos de estudios y Estado de ánimo Ansioso que los estudiantes del Perfil 1, Engagement puro.

Asimismo, nuevamente, el estudio detallado de los efectos post-hoc, y particularmente del tamaño del efecto, permitió contrastar la hipótesis relativa al efecto del Engagement sobre la Procrastinación mediante las comparaciones entre el Perfil 2 y el Perfil 3, pudiéndose observar efectos de amortiguación en tres de las variables consideradas, Persistencia, Evitación de las Dificultades y Desinterés.

\section{Discusión}

Los objetivos del presente estudio pretendían dar respuesta a tres preguntas: 1) ¿están la procrastinación y el Engagement académicos inversamente relacionados?; 2) ¿qué patrón de funcionamiento siguen ambos en relación a los procesos de aprendizaje?; y 3) ¿podría producirse un efecto de amortiguación de los efectos nocivos de la Procrastinación Académica en los estudiantes procrastinadores con niveles de engagement medio-altos?

Los resultados hallados confirman que las variables Procrastinación académica y Engagement se relacionan de manera inversa y moderada.

En cuanto a las relaciones que la Procrastinación Académica y el Engagement presentan con el resto de las variables, es interesante resaltar que algunas variables correlacionan de forma elevada tanto con la Procrastinación Académica como con el Engagement, pero en sentido opuesto. Así, la Persistencia, la Percepción de buena preparación para los exámenes, el factor de Autorregulación Eficiente y dos variables del estado previo a exámenes, Desinterés y Estado de Ánimo Positivo, muestran una relación potente e inversa con los dos constructos objeto de estudio.

Se confirma, asimismo, la asociación de la Procrastinación Académica con variables que obstaculizan el proceso de estudio, como son la Autorregulación Deficiente, las Dificultades atribuidas a falta de tiempo para el estudio y la relación inversa con la Percepción de una buena preparación para el examen. Especialmente potente es la correlación de la Procrastinación Académica con el Manejo ineficaz del tiempo indicando que esta última es la estrategia de actuación propia de los procrastinadores. Estos datos son similares a los informados, entre otros, por Lay y Schouwenburg (1993) o Schouwenburg (2004) quienes mostraron que la procrastinación está inversamente relacionada con estrategias de aprendizaje que impliquen una aproximación sistemática y disciplinada que exija planificación y un manejo del tiempo adecuado.

El Engagement, por el contrario, se relaciona, si bien de forma más moderada, con sentirse mejor preparado para los exámenes, con un estado de ánimo positivo y con un mayor interés. Estos resultados son congruentes con los estudios que informan que emociones 
positivas como la felicidad, el interés y la activación son indicadores de engagement emocional en los estudiantes (Lyubomirsky, King y Diener, 2005; Skinner y Belmont, 1993). Al tiempo, tomados en su conjunto con los datos relativos a las estrategias de autorregulación utilizadas, permiten aventurar que estas estrategias podrían haber producido un efecto beneficioso sobre el sentirse bien preparado/a para los exámenes, lo que, a su vez, podría estar asociado con el incremento de emociones positivas experimentado. Quedaría por analizar si, de igual forma, se habría producido una relación positiva con el rendimiento académico objetivo, tal y como la evidencia empírica del impacto del Engagement sobre el logro académico muestra (Finn y Zimmer, 2012).

Estos resultados corroboran lo referido en otras investigaciones realizadas con población similar acerca de la existencia de dos perfiles antagónicos de afrontamiento estratégico de los estudios (Sánchez-Elvira-Paniagua et al., 2006a,b) vinculándose el perfil positivo con el Engagement y el negativo con la Procrastinación Académica (Sánchez-Elvira-Paniagua, Lisbona, González-Brignardello, Palací y LópezGonzález, 2012).

Estos resultados son replicados, de forma más potente, en los análisis realizados entre Perfiles, especialmente entre los perfiles de Engagement puro y Procrastinación Académica pura; es decir, los grupos extremos. Estos dos perfiles difieren significativamente entre sí en prácticamente todas las variables consideradas, a excepción del uso de estrategias de aprendizaje superficial, lo que es congruente con el estudio factorial previo que indica que este tipo de estrategias pueden considerarse tanto en el factor global de estrategias de autorregulación del aprendizaje eficientes, como en el factor de estrategias deficientes (Sánchez-Elvira-Paniagua et al., 2006b).

El estudio arroja, por tanto, resultados similares a los estudios previos que informan que los procrastinadores no hacen uso de estrategias de aprendizaje efectivas y, con frecuencia, carecen de habilidades y cono- cimientos sobre el uso de estrategias metacognitivas (Howell y Watson, 2007; Wolters, 2003), caracterizándose, asimismo, por presentar escasas habilidades de manejo del tiempo y desorganización (Howel y Watson, 2007; Steel, 2007). Por el contrario, en lo que se refiere al Engagement, cabe destacar la relación positiva encontrada con el uso de estrategias eficientes de autorregulación del aprendizaje, fundamentalmente con Persistencia, confirmando el carácter favorecedor del rendimiento óptimo en entornos académicos que el Engagement presenta, defendido por la Psicología Positiva en el ámbito educativo, así como el carácter propositivo que le otorgan otros autores, en la medida que se concibe como la causa de actos de implicación voluntaria y propositiva en actividades de aprendizaje (Coates, 2005). Asimismo, los datos apuntan en la dirección de que el Engagement y la autorregulación del aprendizaje son constructos que comparten procesos cognitivos, motivacionales y emocionales importantes (Wolters y Taylor, 2012).

Una vez comprobado que los Perfiles 1 y 3 difieren en prácticamente todas las variables consideradas, el estudio detallado de los efectos del Engagement sobre la procrastinación se ha realizado mediante la comparación de las diferencias halladas, entre el Perfil 2 y los otros dos perfiles. Así, cuando el Perfil 2 se diferencia significativamente del Perfil 3, aproximándose al Perfil 1 con un tamaño del efecto al menos de valor medio, hemos considerado que el Engagement produce un efecto de amortiguación de los efectos nocivos de la procrastinación; si el tamaño del efecto es pequeño, se ha estimado una mejora leve, aunque no haya diferencias significativas; en aquellos casos en los que el Perfil 2 no difiere del Perfil 1 se considera que el Engagement produce una equiparación, mientras que en los casos en los que el Perfil 2 y el 3 no difieren, se puede concluir que el Engagement no ejerce un efecto beneficioso sobre los efectos de la procrastinación. La tabla 6 resume los resultados obtenidos, en función del tamaño del efecto. 
Tabla 6

Resumen de efectos encontrados en la comparativa P1/P2 y P2/P3

\begin{tabular}{|c|c|c|c|c|}
\hline & Amortiguación & Equiparación & Leve mejora & Sin cambio \\
\hline \multicolumn{5}{|l|}{ Factores globales de autorregulación } \\
\hline Autorregulación eficiente & 0 & & & \\
\hline Autorregulación deficiente & & & & 0 \\
\hline \multicolumn{5}{|l|}{ Estrategias y hábitos eficientes } \\
\hline Persistencia & 0 & & & \\
\hline Procesamiento profundo & & 0 & & \\
\hline Pautas Fijas de Estudio & & & 0 & \\
\hline Organiz. de la Informac. & & & 0 & \\
\hline Procesamiento superficial & & & 0 & \\
\hline \multicolumn{5}{|l|}{ Estrategias y hábitos deficientes } \\
\hline Manejo Ineficaz del tiempo & 0 & & & \\
\hline Evitación de dificultades & 0 & & & \\
\hline Ansiedad ante estudios & & & & 0 \\
\hline \multicolumn{5}{|l|}{ Estado positivo } \\
\hline Percepción de buena preparación & 0 & & & \\
\hline Estado de ánimo Positivo & 0 & & & \\
\hline \multicolumn{5}{|l|}{ Estado negativo } \\
\hline Dificultades atribuidas a falta tiempo & & & & 0 \\
\hline Desinterés & 0 & & & \\
\hline $\begin{array}{l}\text { Interferencias por falta de hábitos } \\
\text { de estudio }\end{array}$ & & & 0 & \\
\hline Estado de ánimo Ansioso & & & & 0 \\
\hline
\end{tabular}

Nota. Organiz. de la Informac. (Organización de la Información)

Los perfiles de clasificación presentan un comportamiento diferencial ante las variables del estudio, de tal modo que se confirma, en general, el efecto buffering o de amortiguación del Engagement sobre los efectos de la procrastinación:

- A mayores niveles de Engagement, mayor utilización de estrategias de autorregulación efectivas y disminución de los deficientes. Así, el aumento de los niveles de Engagement parece producir en el Perfil de Procrastinadores con Engagement medio-alto, Perfil 2, un claro efecto de amortiguación en siete de las variables consideradas, incrementando significativamente los niveles relativos a la utilización de estrategias de autorregulación eficientes, globalmente consideradas $\mathrm{y}$, específicamente, la persistencia y el estado de ánimo positivo previo a los exámenes. Asimismo, a mayores niveles de Engagement, menor utilización de estrategias y hábitos deficientes, como manejo ineficaz del tiempo, evitación de dificultades y desinterés.

- El efecto positivo lleva a equiparar a los Perfiles 1 y 2, en el uso de estrategias de aprendizaje profundo.

- Asimismo, se observa una mejoría leve en el Perfil 2 en el uso de las estrategias de aprendizaje superficial. Tenien- 
do en cuenta que también incrementan el uso de estrategias profundas, estaría indicando que emplean mejores estrategias de aprendizaje, en conjunto. Este efecto leve también se observa en la disminución de la percepción de interferencias por falta de hábitos de estudio, en el incremento de pautas fijas de estudio y en organización de la información.

- Finalmente, los Procrastinadores con Engagement medio-alto (P2) no se ven beneficiados por los mayores niveles de Engagement, es decir, no presentan diferencias con el Perfil de Procrastinadores puros (P3) en cuanto a los niveles de ansiedad informados durante el periodo de estudio y antes de los exámenes, o en su percepción de haber experimentado interferencias por la falta de tiempo para el estudio, difiriendo significativamente del Perfil 1.

Es importante, resaltar los efectos de amortiguación y mejora encontrados en el perfil de procrastinadores con niveles medio-altos de Engagement, especialmente sobre dos aspectos comportamentales centrales a la Procrastinación, como son la falta de persistencia y el manejo ineficaz del tiempo, siendo especialmente importantes los efectos de la primera. En ese sentido, los resultados de este estudio, en cuanto al comportamiento de sujetos que, aún siendo procrastinadores, presentan niveles de engagement medio-altos, parecen estar en relación con recientes hallazgos acerca de la existencia de un perfil de "procrastinadores activos», que no sufre las consecuencias nefastas de la procrastinación pasiva. Estos procrastinadores escogen voluntariamente retrasar la realización o finalización de la tarea, persiguiendo estados de alta concentración y ejecución bajo la presión del deadline y que se asocia con variables psicológicas y resultados académicos positivos (Choi y Morán, 2009; Chu y Choi, 2005; Seo, 2012). Además, en nuestra muestra, los sujetos del perfil 2 se diferenciaron, especialmente, por presentar un estado de ánimo más positivo, se percibieron mejor preparados y se mostraron más interesados antes de los exámenes que los procrastinadores puros.
Por otro lado, la equiparación en el uso de estrategias de aprendizaje profundo entre los estudiantes con niveles elevados de engagement, y los procrastinadores con engagement medio-alto, sugiere el claro efecto beneficioso del engagement para los procrastinadores, en la medida en que, por un lado, la procrastinación ha sido vinculada a la menor utilización de estrategias cognitivas y meta cognitivas de aprendizaje (Howell y Watson, 2007) y, por otro, el engagement cognitivo al procesamiento profundo o significativo (Walker, Greene y Mansell, 2006).

Este estudio abre interrogantes para futuras investigaciones acerca de cómo promover el engagement en los estudiantes procrastinadores así como el tipo de estrategias de autorregulación del aprendizaje que podrían entrenarse, sabiendo que unas serán más flexibles y modificables que otras, de cara a establecer modelos de intervención más efectivos sobre la procrastinación académica con el objetivo último de prevenir el abandono y el fracaso de los estudiantes. En este marco, y continuando con un formato parecido al de este estudio, se plantea, asimismo, la posibilidad de analizar los efectos de otras variables positivas relacionadas con el Engagement, como los factores motivacionales (Reeve, 2012), la resiliencia, la esperanza o el optimismo (Brooks et al., 2012).

Los resultados de este estudio ayudan a entender la procrastinación, los posibles tipos de procrastinadores y las estrategias utilizadas; sin embargo, presenta algunas limitaciones que han de ser tomadas en cuenta en futuras investigaciones. En este estudio hemos utilizado un cuestionario experimental que evalúa diferentes aspectos del proceso de estudio a lo largo de un cuatrimestre y que no ha sido estandarizado y, por lo tanto, presta una utilidad exploratoria. Por otra parte, los datos provienen exclusivamente de autoinformes, debiendo añadirse en un futuro variables observacionales o de rendimiento que permitan ampliar la validez de las conclusiones acerca de las implicaciones que tiene la procrastinación y el Engagement en este ámbito de educación superior a distancia. 


\section{Referencias}

Ariely, D. y Wertenbroch, K. (2002). Procrastination, deadlines, and performance: Self-Control by precommitment. Psychological Science, 13, 219224. http://dx.doi.org/10.1111/1467-9280.00441

Beswick, G., Rothblum, E. D. y Mann, L. (1988). Psychological Antecedents of Student Procrastination. Australian Psychologist, 23, 207-217. http://dx.doi.org/10.1080/00050068808255605

Bisward-Diener, R. y Dean, B. (2005). Positive Psychology Coaching. Putting the science of happiness to work for your clients. New Jersey, NJ.: Wiley.

Blunt, A. K. y Pychyl, T. A. (2000). Task aversiveness and procrastination: a multi-dimensional approach to task aversiveness across stages of personal projects. Personality and Individual Differences, 28, 153-167. http://dx.doi.org/10.1016/ S0191-8869(99)00091-4

Brooks, R., Brooks, S. y Godstein, S. (2012). The Power of mindsets: nurturing engagement, motivation and resilience in students. En S.L. Christenson, A.L., Reschly y C. Cathy (Eds.), Handbook of research on student engagement (pp. 541-562). New York, NY.: Springer.

Bruinsma, M. y Jansen, E. P.W.A.(2009). When will I succeed in my first-year diploma? Survival analysis in Dutch higher education. Higher Education Research \& Development, 28, 99-114. doi: 10.1080/072943608024443

Choi, J. M. y Morán, S.V. (2009). Why not procrastinate? Development and validation of a new Active Procrastination Scale. The Journal of Social Psychology, 149, 195-211.

Christenson, S., Reschly, A. L. y Wylie, C. (2012). Epilogue. En S. L. Christenson, A. L. Reschly y C. Wylie (Eds.), Handbook of research on student engagement (pp. 813-818). New York, NY.: Springer.

Chu, A. H. C. y Choi, J. N. (2005). Rethinking procrastination: Positive effects of «active» procrastination behavior on attitudes and performance. Journal of Social Psychology, 145, 245-264.

Coates, H. (2005). The Value of Student Engagement for Higher Education Quality Assurance. Quality in Higher Education, 11(1), 25-36. doi: 10.1080/02602930600801878
Cohen, J. (1988). Statistical Power Analysis for the Behavioral Sciences (2. ${ }^{\mathrm{a}}$ Ed.). Hillsdale, NJ.: Erlbaum.

Csikszentmihalyi, M. (1996). Fluir (Flow). Una psicología de la felicidad [Flow. The psychology of optimal experience]. Barcelona, España: Kairós.

Ellis, P. D. (2009). Effect size calculators [Página web]. Recuperado febrero 10, 2013, de http:// www.polyu.edu.hk/mm/effectsizefaqs/calculator/ calculator.html

Ferrari, J. R. (2010). Still Procrastinating?. The noregrets guide to getting it done. New Jersey, NJ.: Wiley.

Ferrari, J. R. y Tice, D. M. (2000). Procrastination as a Self-Handicap for men and women: A task-avoidance strategy in a laboratory setting. Journal of Research in Personality, 34, 73-83. doi:10.1006/jrpe.1999.2261

Ferrari, J. R., Parker, J. T. y Ware, C. B. (1992). Academic procrasti-nation: Personality correlates with Myers-Briggs types, self-efficacy, and academic locus of control. Journal of Social Behavior and Personality, 7, 495-502.

Finn, J. D. y Zimmer, K. S. (2012). Student engagement: What is it? Why does it matter? En S. L. Christenson, A. L. Reschly y C. Wylie (Eds.), Handbook of research on student engagement (pp. 97-132). New York, NY.: Springer.

Freuderberger, H. J. (1974). Staff burn-out. Journal of Social Issues, 30, 159-165.

González-Brignardello, M. P. y Sánchez-ElviraPaniagua, A. (n.d.). Procrastinación Académica - cuestionario experimental (manuscrito sin publicar).

Harriott, J. y Ferrari, J. R. (1996). Prevalence of Procrastinations among samples of adults. Psychological Reports, 78, 611-616. http://dx.doi. org/10.2466/pr0.1996.78.2.611

Howell, A. J. y Watson, D. C. (2007). Procrastination: Associations with achievement goal orientation and learning strategies. Personality and Individual Differences, 43, 167-178. doi:10.1016/j.paid.2006.11.017

Howell, A. J., Watson, D. C., Powell, R. A. y Buro, K. (2006). Academic procrastination: The pattern and correlates of behavioural postponement. Personality and Individual Differences, 40, 15191530. doi:10.1016/j.paid.2005.11.023 
Lay, C. H. y Silverman, S. (1996). Trait procrastination, anxiety, and dilatory behavior. Personality and Individual Differences, 21, 61-67. http:// dx.doi.org/10.1016/0191-8869(96)00038-4

Lay, C. y Schouwenburg, H. (1993). Trait procrastination, time management, and academic behavior. Journal of Social Behavior and Personality, $8,647-662$.

Lyubomirsky, King y Diener, (2005). The Benefits of Frequent Positive Affect: Does Happiness Lead to Success? Psychological Bulletin, 131, 803-855.

Macan, T. H. (1996). Time-Management Training: Effects on Time Bejaviors, Attitudes, and Job Performance. The Journal of Psychology, 130, 229-236. http://dx.doi.org/10.1080/00223980.19 96.9915004

Martínez, I. M. y Salanova, M. (2003). Niveles de Bournout y Engagement en estudiantes universitarios. Relación con el desempeño y desarrollo profesional. Revista de Educación, 330, 361-384.

Montero, I. y León, G. (2007). A guide for naming research studies in Psychology. International Journal of Clinical and Health Psychology, 7, 847-862.

Özer, B. U., Demir, A. y Ferrari, J. P. (2009). Exploring Academic Procrastination among Turkish Students: Possible Gender Differences in Prevalence and reasons. The Journal of Social Psychology, 149, 241-257. http://dx.doi.org/10.3200/ SOCP.149.2.241-257

Park, S. W. P. y Sperling, R. A. (2012). Academic Procrastination and their Self-Regulation. Psychology, 3, 12-23. doi:10.4236/psych.2012.31003

Peterson, Ch. y Seligman, M. E. P. (2004). Character strengths and virtues: a handbook and classification. New York, NY: Oxford University.

Pychyl, T. A., Lee, J. M., Thibodeau, R. y Blunt, A. (2000). Five Days of Emotion: An Experience Sampling Study of Undergraduate Student Procrastination. Journal of Social Behavior and Personality, 15 [Special Issue], 239-254.

Reeve, J. (2012). A Self-determination Theory perspective on student engagement. En S. L. Christenson, A. L. Reschly y C. Cathy (Eds.), Handbook of research on student engagement (pp. 3-20). New York, NY.: Springer, 149-172.
Reschly, A. L. y Christerson, S. L. (2012). Jingle, jangle, and conceptual haziness: evolution and future directions of engagement construct. En S. L. Christenson, A. L. Reschly y C. Cathy (Eds.), Handbook of research on student engagement (pp. 3-20). New York, NY.: Springer.

Rosario, P., Núñez, J. C., Salgado, A., GonzálezPienda, J. A., Valle, A., Joly, C. y Bernardo, A. (2008). Ansiedad ante los exámenes: Relación con variables personales y familiares. [Test anxiety: Relationship to personal and family variables]. Psicothema, 20, 563-570.

Rosenthal, J. A. (1996). Qualitative descriptors of strength of association and effect size. Journal of Social Service Research, 21(4), 37-59.

Rothblum, E. D., Solomon, L. J. y Murakami, J. (1986). Affective, Cognitive, and Behavioral Differences between High and Low Procrastinators. Journal of Counseling Psychology, 33, 387-394. http://dx.doi. org/10.1037//0022-0167.33.4.387

Salanova, M., Schaufeli, W. B., Llorens, S., Peiró, J. M. y Grau, R. (2000). Desde el «burnout»al «Engagement»: ¿una nueva perspectiva? [From the «burnout» to «Engagement»: A new perspective?]. Revista de Psicología del Trabajo y de las Organizaciones, 16, 117-134.

Sánchez-Elvira-Paniagua, A. y González-Brignardello, M. P. (n.d.). Batería experimental elaborada ad hoc para el Plan de Acogida Virtual UNED Fase de preparación de exámenes-cuestionario experimental (manuscrito sin publicar).

Sánchez-Elvira-Paniagua, A., Fernández, E. y Amor, P. (2006a). Self-regulated learning in distance education students: preliminary data. En A. Delle Fave (Ed.), Dimensions of Well-being: Research and Intervention, (pp. 294-314). Milan, Roma: FrancoAngeli.

Sánchez-Elvira-Paniagua, A., Fernández, E. y Amor, P. (2006b, julio). Predictive power of efficient vs. non efficient self regulated learning strategies, general vs. specific personality variables and life events on stress, well-being and academic satisfaction among distance education students. Actas de Congreso de la 13th European Conference on Personality (p. 216). Atenas, Grecia.

Sánchez-Elvira-Paniagua, A., González-Brignardello, M. P. y Santamaría-Lancho. M. (2009, junio). The benefits of the use of Induction Vir- 
tual Communities in supporting new students in distance education universities. ICDE 2009. Maastrich, Países Bajos. Recuperado de http:// www.ou.nl/Docs/Campagnes/ICDE2009/Papers/ Final_paper_266Sanchez-Elvira.pdf

Sánchez-Elvira-Paniagua, A., Lisbona, A., GonzálezBrignardello, M., Palací, F. y López-González, M. A. (2012, marzo). Recursos personales de los estudiantes universitarios y su relación con el desempeño académico y el bienestar personal [Personal resources of university students and its relationship to academic performance and personal wellbeing]. I Congreso de Psicología Positiva Española. San Lorenzo de El Escorial, SEPP.

Schaufeli, W. B., Martínez, I. M., Marques Pinto, A., Salanova, M. y Bakker, A. B. (2002). Bournout and Engagement in university students. Journal of Cross-Cultural Psychology, 33, 464-481.

http://dx.doi.org/10.1177/0022022102033005003

Schaufeli, W. B., Salanova, M., González-Romá, V. y Bakker, A. B. (2002). The measurement of Engagement and burnout: a two simple confirmatory factor analytic approach. Journal of Happiness Studies, 3, 71-92.

Schouwenburg, H. C. (2004). Procrastination in academic settings: general introduction. En $\mathrm{H}$. C. Schouwenburg, C. H. Lay, T. A. Pychyl y J. R. Ferrari (Eds.), Counseling the procrastinator in academic settings (pp. 3-17). Washington, DC.: American Psycological Association.

Seligman, M. E. P. y Csikszentmihalyi, M. (2000). Positive psychology: An introduction. American Psychologyst, 55, 5-14. http://dx.doi. org/10.1037//0003-066X.55.1.5

Semb, G., Glick, D. M. y Spencer, R. E. (1979). Student withdrawals and delayed work patterns in Self-Paced Psychology Courses. Teaching of Psychology, 6, 23-25. http://dx.doi.org/10.1207/ s15328023top0601_8

Seo, E. H. (2012). Cramming, active procrastination, and academic achievement. Social Behavior and Personality, 40, 1333-1340. http://dx.doi. org/10.2224/sbp.2012.40.8.1333
Skinner, E. A. y Belmont, M. J. (1993). Motivation in the classroom: Reciprocal effects of teacher behavior and student engagement across the school year. Journal of Educational Psychology, 85, 571-581. http://dx.doi.org/10.1037//00220663.85 .4 .571

Solomon, L. J. y Rothblumn, E. D. (1984). Academic Procrastination: frequency and cognitive-behavioral correlates. Journal of Counseling Psychology, 31, 503-509. http://dx.doi. org/10.1037//0022-0167.31.4.503

Steel, P. (2007). The nature of Procrastination: A Meta-analytic and theoretical review of quintessential self-regulatory failure. Psychological Bulletin, 133, 65-94. doi: 10.1037/00332909.133.1.65

Sun, J. C. -Y. y Rueda, R. (2012). Situational interest, computer self-efficacy and self-regulation: Their impact on student engagement in distance education. British Journal of Educational Technology, 2, 191-204. doi:10.1111/j.14678535.2010.01157.x

Van Eerde, W. (2003). A meta-analytically derived nomological network of procrastination. Personality and Individual Differences, 35, 1401-1418.

Walker, C. O., Greene, B. A. y Mansell, R. A. (2006). Identification with academics, intrinsic/extrinsic motivation, an self-efficacy as preditors of cognitive engagement. Learning and individual Differences, 16, 1-12. doi: 10.1016/j.lindif.2005.06.004

Wolters, C. A. (2003). Understanding Procrastination from Self-regulated learning perspective. Journal of Educational Psychology, 95, 179-187. http://dx.doi.org/10.1037//0022-0663.95.1.179

Wolters, Ch. y Taylor, D. J. (2012). A self-regulated learning perspective on student engagement. En S. L. Christenson, A. L. Reschly y C. Wylie (Eds.), Handbook of research on student engagement (pp. 635-651). New York, NY.: Springer. 


\section{Apéndice}

Tabla 1

Fase de aplicación, Instrumento, Variables y sus descriptivos

\begin{tabular}{|c|c|c|c|c|}
\hline Fase & Instrumento & Variable & Media & DT \\
\hline \multirow{12}{*}{ Estudio } & $\begin{array}{l}\text { Procrastinación } \\
\text { Académica }\end{array}$ & Procrastinación Académica & 2.29 & .65 \\
\hline & \multirow{3}{*}{ Engagement } & Engagement Absorción & 4.11 & .95 \\
\hline & & Engagement Vigor & 4.06 & 1.04 \\
\hline & & Engagement Dedicación & 5.06 & .85 \\
\hline & \multirow{8}{*}{$\begin{array}{c}\text { Estrategias de aprendizaje } \\
\text { autorregulado y hábitos } \\
\text { de estudio }\end{array}$} & Ansiedad ante los estudios & 1.90 & .64 \\
\hline & & Persistencia & 3.46 & .45 \\
\hline & & Manejo ineficaz del tiempo & 2.17 & .58 \\
\hline & & Procesamiento Profundo & 2.95 & .53 \\
\hline & & Organización de la Información & 3.20 & .65 \\
\hline & & Procesamiento Superficial & 2.86 & .60 \\
\hline & & Evitación de Dificultades & 1.48 & .43 \\
\hline & & Pautas Fijas de Estudio & 3.53 & .55 \\
\hline \multirow{7}{*}{$\begin{array}{l}\text { Estado } \\
\text { previo } \\
\text { a los } \\
\text { exámenes }\end{array}$} & \multirow{3}{*}{$\begin{array}{c}\text { Percepción } \\
\text { de preparación, } \\
\text { dificultades e interfirientes }\end{array}$} & Atribución Falta de tiempo & 2.32 & .70 \\
\hline & & $\begin{array}{l}\text { Percepción de Buena Preparación para los } \\
\text { exámenes }\end{array}$ & 2.94 & .69 \\
\hline & & Interferencias por falta de hábitos de estudio & 2.18 & .98 \\
\hline & \multirow{4}{*}{ Estado de ánimo } & Estado de Ánimo Positivo & 2.76 & .79 \\
\hline & & Estado de Ánimo Ansioso & 2.20 & .80 \\
\hline & & Desinterés & 1.35 & .50 \\
\hline & & Estado Somático-corporal & 3.65 & .82 \\
\hline
\end{tabular}

